\title{
Relación de glutatión reducido/oxidado (GSH/GSSG) en ratas diabéticas tratadas con maca (Lepidium meyenii walp)
}

Value of reduced / oxidized glutathione (GSH / GSSG) in diabetic rats treated with maca

(Lepidium meyenii Walp)

\author{
Ruth Cisneros' ${ }^{1}$, Raquel Oré2, Inés Arnao², Silvia Suárez² \\ Docente, Departamento de Ciencias Dinámicas, Facultad de Medicina, Universidad Nacional Mayor de San Marcos. Lima, Perú. \\ ${ }^{2}$ Centro de Investigación de Bioquímica y Nutrición, Facultad de Medicina, Universidad Nacional Mayor de San Marcos. Lima, Perú.
}

\begin{abstract}
Resumen
Introducción: La diabetes mellitus y sus complicaciones representan un problema de salud pública. La hiperglucemia desencadena efectos bioquímicos dañinos para el organismo, como el estrés oxidativo y el proceso inflamatorio crónico, por lo que el uso de plantas medicinales con elevada capacidad antioxidante, como Lepidium meyenii Walp (maca), es una alternativa en el tratamiento de esta patología. Objetivo: Determinar si la ingestión de harina de maca mejora la relación glutatión reducido/oxidado (GSH/GSSG) en ratas diabéticas. Diseño: Experimental. Institución: Centro de Investigación de Bioquímica y Nutrición, Facultad de Medicina, UNMSM, Lima, Perú. Material biológico: Harina de maca amarilla y ratas albinas Holtzman machos adultas. Intervenciones: Se utilizó 24 ratas, a los cuales se les indujo la diabetes con estreptozotocina (STZ) $40 \mathrm{mg} / \mathrm{kg}$ de masa corporal y que fueron distribuidas aleatoriamente en cuatro grupos, de seis animales cada uno: control (CO), glibenclamida (GL) $10 \mathrm{mg} / \mathrm{kg}$ de masa corporal, maca $4 \mathrm{~g} / \mathrm{dia}(\mathrm{M} 4)$ y maca $6 \mathrm{~g} / \mathrm{dia}$ (M6); el experimento duró 40 días. Se evaluó la glicemia, masa corporal y, al final del experimento, se determinó los niveles de GSH y GSSG en plasma y homogenizado de hígado. Principales medidas de resultados: Masa corporal, glicemia, GSH y GSSG en plasma y homogenizado de hígado. Resultados: A nivel plasmático, el glutatión circulante estuvo favorecido para la forma reducida. Los valores de la relación GSH/GSSG en los grupos tratados con maca estuvieron alrededor de 190, significativamente mayor al grupo CO. Conclusiones: La administración de harina de maca amarilla a ratas diabéticas incrementó la relación GSH/GSSG a nivel plasmático, contribuyendo a mejorar su estado redox.
\end{abstract}

Palabras clave: Diabetes, maca amarilla, glutatión reducido, glutatión oxidado, estrés oxidativo.

\section{Abstract}

Background: Diabetes mellitus and its complications are public health problems. Hyperglycemia triggers harmful biochemical effects to the body, such as oxidative stress and inflammatory process. The use of medicinal plants with high antioxidant capacity as Lepidium meyenii Walp (maca) is an alternative in treatment of this disease. Objective: To determine whether ingestion of maca flour improves the relation reduced/oxidized glutathione (GSH/GSSG) in diabetic rats. Design: Experimental. Setting: Biochemistry and Nutrition Research Center, Faculty of Medicine, Universidad Nacional Mayor de San Marcos, Lima, Peru. Biological material: Yellow maca flour and adult male Holtzman albino rats. Interventions: Diabetes was induced in 24 rats with streptozotocin (STZ) $40 \mathrm{mg} / \mathrm{kg}$ body mass; rats were randomly divided into four groups of six animals each: control (CO), glibenclamide (GL) $10 \mathrm{mg} / \mathrm{kg}$ body mass, maca $4 \mathrm{~g} / \mathrm{day}$ (M4), and maca $6 \mathrm{~g} /$ day (M6). The experiment lasted 40 days. We evaluated glucose, body mass and levels of GSH and GSSG in plasma and liver homogenate at the end of the experiment. Main outcome measures: Body mass, plasma glucose, GSH and GSSG in plasma and liver homogenate. Results: Plasma was favored with circulating reduced glutathione. Values of GSH / GSSG ratio in groups treated with maca were about 190, significantly higher than in the CO group. Conclusions: Yellow maca flour administration to diabetic rats increased the GSH / GSSG ratio in plasma and contributed in improving redox status.

Key words: Diabetes, yellow maca, reduced glutathione, oxidized glutathione, oxidative stress.

An Fac med. 2011;72(2):107-11

\section{INTRODUCCIÓN}

La diabetes mellitus (DM) es una enfermedad crónica de elevada incidencia y prevalencia a nivel mundial, constituyendo en la actualidad uno de los graves problemas de salud pública ${ }^{(1,2)}$. En el Perú, la prevalencia de diabetes es de 1 a $8 \%$ en la población general, encontrándose Piura y Lima como los más afectados ${ }^{(3)}$. En la actualidad, afecta a más de un millón de peruanos y menos de la mitad ha sido diagnosticado ${ }^{(4)}$. La diabetes mellitus es una enfermedad metabólica de origen endocrino, cuya principal característica bioquímica es la hiperglucemia crónica asociada a fallas en la acción o producción de la insulina, con alteraciones del metabolismo intermedio de lípidos y proteínas ${ }^{(5)}$. En lo que se refiere a la investigación sobre DM, se ha desarro- llado diversas líneas que pretenden explicar los mecanismos fisiopatológicos y complicaciones en esta enfermedad. Se ha señalado de manera consistente a la hiperglucemia crónica como la condición fisiológica responsable del desarrollo de las complicaciones del paciente diabético, a través de diversos mecanismos bioquímicos que llevan a procesos como el estrés oxidativo y la inflamación crónica ${ }^{(5)}$. 
En nuestro organismo existen diferentes mecanismos para evitar la extensión del daño causada por los radicales libres. Estos incluyen sistemas enzimáticos y moléculas exógenas y endógenas, como el glutatión reducido (GSH), metabolito empleado como parámetro de estrés oxidativo para evaluar implicancias clínicas, integridad y funcionalidad ${ }^{(6)}$. Esta biomolécula es un tripéptido (L-y-glutamil-L-cisteinilglicina) y tiene un papel fundamental en la protección celular contra la injuria oxidativa. Tiene un enlace $\gamma$-glutamilo y un grupo tiol libre. El primer enlace impide que el GSH sea hidrolizado por las $\alpha$-peptidasas. El segundo hace que el GSH sea extraordinariamente reactivo con otras sustancias, incluyendo xenobióticos ${ }^{(7)}$.

Los cambios de concentración de GSH en sangre podrían dar una medida del estrés oxidativo in vivo ${ }^{(8)}$. La importancia de sus funciones dentro de los sistemas biológicos ha dado lugar a la existencia de un sistema GSH que incluye además enzimas relacionadas con su metabolismo, responsables del mantenimiento de su estado redox en condiciones fisiológicas ${ }^{(9)}$. La oxidación enzimática del GSH se produce como producto de la actividad de la glutatión peroxidasa (GPx), tal como se describe a continuación ${ }^{(10,11)}$.

$$
\mathrm{H}_{2} \mathrm{O}_{2}+2 \mathrm{GSH} \stackrel{\mathrm{GPx}}{\longrightarrow} 2 \mathrm{H}_{2} \mathrm{O}+\underset{\begin{array}{c}
\text { Glutatión } \\
\text { oxidado }
\end{array}}{\mathrm{GSSG}}
$$

El glutatión oxidado (GSSG) es luego reducido por la glutatión reductasa (GRd), que utiliza la coenzima nicotinamida adenina dinucleótido fosfato reducido (NADPH), proveniente de la vía pentosa fosfato como dador de electrones, manteniendo así la proporción GSH/GSSG ${ }^{(12,13)}$.

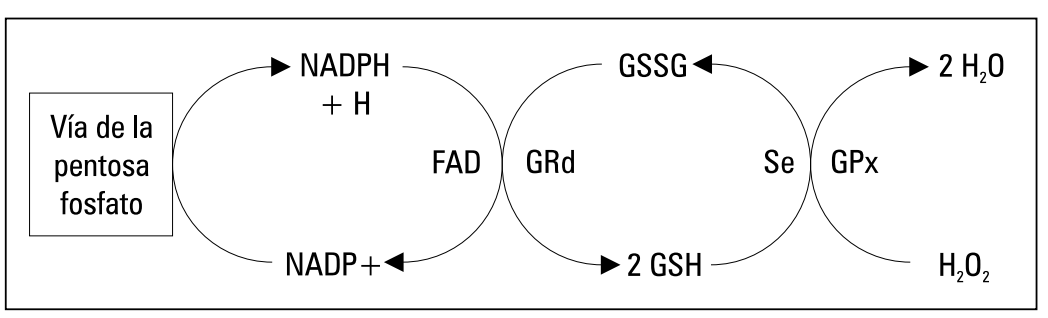

El GSH está sujeto a un constante recambio en el organismo; hígado, riñones, pulmones, corazón, intestinos y músculos son los principales órganos responsables de su homeostasis ${ }^{(14)}$.

Desde hace unas décadas se realizan esfuerzos a nivel mundial para revalorar la medicina tradicional y alternativa para contribuir a mitigar los problemas de salud pública, como la diabetes. En ese esfuerzo, nuestro país ha aportado recursos naturales, como el Lepidium peruvianum variedad Chacón, también conocida como Lepidium meyenii Walp 'maca', que se cultiva principalmente en las regiones Suni y Puna, de los departamentos de Junín y Pasco, entre los 3700 y 4500 m.s.n.m. ${ }^{(15)}$. Su raíz constituye la porción comestible de la planta, y la medicina tradicional le atribuye propiedades afrodisíacas, reguladora, inmunoestimulante, energizante y antiartrítica ${ }^{(16)}$. Entre otros usos, también se la emplea para la reducción de los efectos del estrés y la fatiga. Aún cuando los mecanismos no han sido dilucidados, se ha propuesto que la maca es citostática y antitumoral, antioxidante e hipoglicemiante. Es un adaptógeno, que aumenta la energía, la resistencia y reduce el estrés ${ }^{(17,18)}$. Estudios realizados en ratas, ratones y cobayos, han demostrado las propiedades de la maca sobre el comportamiento sexual y sus efectos positivos sobre la fertilidad ${ }^{(19)}$. Su contenido de metabolitos secundarios cuenta con principios activos, como el derivado bencilado 1,2-dihidro-N-hidroxipiridina, llamado macaridina, las macamidas $\mathrm{N}$-bencil5-oxo-6E,8E octadecadienamida, Nbencil-hexadecanamida, ácido acético, ácido 5-oxo-6E,8E-octadecadienoico y los glucosinolatos ${ }^{(20)}$.

El estudio que presentamos tiene como objetivo evaluar los cambios plasmáticos y en el tejido hepático del GSH total y la relación GSH/GSSG al administrar harina de maca amarilla en dos concentraciones, en un modelo experimental de diabetes.

No hay estudios bioquímicos publicados que incluyan los cambios de la relación GSH/GSSG en organismos que reciben maca en un modelo de diabetes, por lo que nuestro aporte es importante para entender los mecanismos de defensa antioxidante involucrados luego de la administración de maca.

\section{MÉTODOS}

La maca ecotipo amarillo usada en la presente investigación fue colectada en Carhuamayo-Junín. Las raíces de maca, de 5 a $7 \mathrm{~cm}$ de diámetro, enteras, fueron limpiadas, eliminándose las raicillas. Luego, fueron lavadas para retirar el detritus, cortadas en láminas y desecadas en una estufa de aire circulante, entre 37 y $40^{\circ} \mathrm{C}$, durante 48 horas, aproximadamente, hasta masa constante. Después se procedió a la molienda y tamizado, hasta obtener un polvo fino que fue conservado en recipientes de plástico sellados herméticamente, hasta antes de su uso.

Se realizó un estudio de tipo experimental, en el que se usó 24 ratas albinas Holtzman machos adultas, de 3 meses de edad, adquiridas en la Universidad Agraria La Molina, cuyas masas corporales estaban comprendidas entre 280 y $320 \mathrm{~g}$, distribuidos de manera aleatoria en cuatro grupos de 6 animales cada uno.

Los animales fueron mantenidos en el bioterio del Centro de Investigación de Bioquímica y Nutrición, de la Facultad de Medicina, UNMSM, en jaulas individuales, con dieta balanceada y agua ad libitum, temperatura promedio de $22^{\circ} \mathrm{C}$ y un ciclo de luz/oscuridad de $12 / 12$ horas. Fueron pesados diariamente durante el tiempo que duró el estudio. Luego del período de adaptación y de un ayuno de 12 horas, se determinó la glicemia como lectura inicial 
$(107,5 \mathrm{mg} / \mathrm{dL} \pm 16,3)$. Para inducir la diabetes, se inyectó estreptozotocina a los cuatro grupos (STZ, Sigma Chemical Co., St Louis), el cual se diluyó con buffer citrato de sodio $10 \mathrm{mM}$, a $\mathrm{pH}$ 4,5 , inmediatamente antes de su uso y se mantuvo en hielo hasta inyectar 40 $\mathrm{mg} / \mathrm{kg}$ de masa corporal, por vía intraperitoneal ${ }^{(21)}$. Se dosó los niveles de glucosa antes de iniciar el tratamiento (día 1) y cada 7 días, durante 40 días.

Los tratamientos administrados a cada grupo fueron los siguientes: $\mathrm{Al}$ grupo control $(\mathrm{CO})$ se le administró 1 $\mathrm{mL}$ de suero fisiológico por vía orogástrica, los grupos maca 4 (M4) y maca 6 (M6) recibieron en su dieta harina de maca amarilla (4 y $6 \mathrm{~g} /$ día), respectivamente. Al grupo glibenclamida $(\mathrm{Gl})$ se le administró por vía orogástrica glibenclamida (10 mg/kg de masa) disuelta en suero fisiológico.

Se tuvo en cuenta las normas y procedimientos éticos para el manejo de animales de experimentación establecidos internacionalmente ${ }^{(22)}$.

Completado el periodo experimental, los animales fueron sacrificados, previa anestesia. Se tomó muestras de hígado y sangre, para las determinaciones bioquímicas. Se preparó un homogenizado de hígado al 10\%, en buffer fosfato 0,05 M, pH 7,4. Se centrifugó a $754 \mathrm{x}$ g, durante 5 minutos, en una centrífuga marca Sorvall, rotor SS-34, y se trabajó con el sobrenadante. El tejido se mantuvo a $4^{\circ} \mathrm{C}$ durante todo el procesamiento, incluyendo los ensayos bioquímicos. El homogenizado de hígado y el plasma fueron acidificados con ácido tricloroacético al 100\%, para determinar el GSH total y el GSSG. Los niveles de glutatión total fueron determinados utilizando el método de Boyne ${ }^{(23)}$, se determinó en presencia de vitamina $\mathrm{C}$, glioxilato y ácido 2 ditiobisnitrobenzoico (DTNB), midiendo en el espectrofotómetro a $412 \mathrm{~nm}$. El GSSG fue calculado restando el GSH del glutatión total.

Para la determinación de glucosa, se obtuvo las muestras de sangre de la base de la cola de las ratas albinas. La

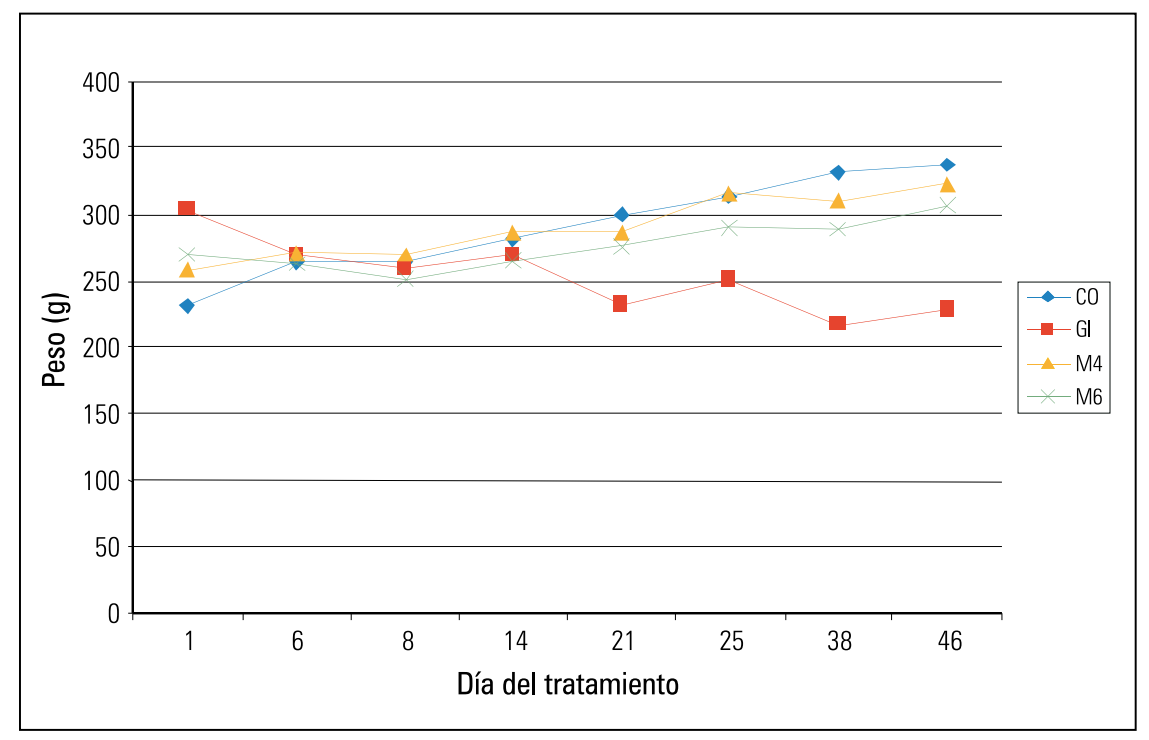

Figura 1. Pesos (g) de ratas diabéticas por día, según grupo de tratamiento.

gota de sangre fue colocada en la tira reactiva del hemoglucotest Accuchec Active de Roche.

Los resultados fueron analizados empleando la prueba t-student; las diferencias han sido consideradas significativas cuando $\mathrm{p}<0,05$.

\section{RESULTADOS}

La figura 1 muestra las masas corporales de todos los grupos, observándose una disminución de masa en el grupo Gl.

La figura 2 señala el porcentaje de disminución de la glicemia a los cuarenta días de tratamiento,observándose que esta fue mayor en el grupo M6.

El porcentaje de disminución de la glicemia a los cuarenta días de tratamiento fue mayor en el grupo M6 $(57,8 \%)$, que en los grupos CO $(27,9 \%)$, GI $(35,2 \%)$ y M4 $(34,4 \%)$.

La administración de la harina de maca 4 y $6 \mathrm{~g} /$ día produjo un incremento de la GSH en el homogenizado de hígado. Lo mismo se observó para el grupo diabético tratado con glibenclamida. Sin embargo, los grupos que recibieron
4 y 6 g/día de harina de maca no mostraron diferencia significativa $(\mathrm{p}<0,05)$ respecto al grupo control (figura 2).

También se observó un incremento de la GSH en el plasma de ambos grupos de ratas tratadas con harina de maca siendo en este caso la diferencia significativa $(p<0,05)$ respecto al grupo control (figura 3).

En las ratas diabéticas que recibieron tratamiento con glibenclamida, se observó claramente cómo estaba incrementado el GSSG en el homogenizado del hígado (figura 2). Sin embargo, en el plasma (figura 3) se observó que el GSSG se encontraba incrementado en el grupo control diabético respecto al grupo tratado con glibenclamida $(\mathrm{p}<0,05)$.

$\mathrm{Al}$ determinar la relación GSH/ GSSG en el homogenizado de hígado (figura 2), se encontró que el grupo M6 tuvo un valor mucho más bajo (30) respecto a los grupos CO, Gl y M4 $(35,37$ y 34 , respectivamente). Sin embargo, en el plasma se encontró que la relación GSH/GSSG fue más baja en el grupo control (137) con respecto a los grupos tratados con maca (M4:191 y M6:188) y glibenclamida (189) (figura 3). 


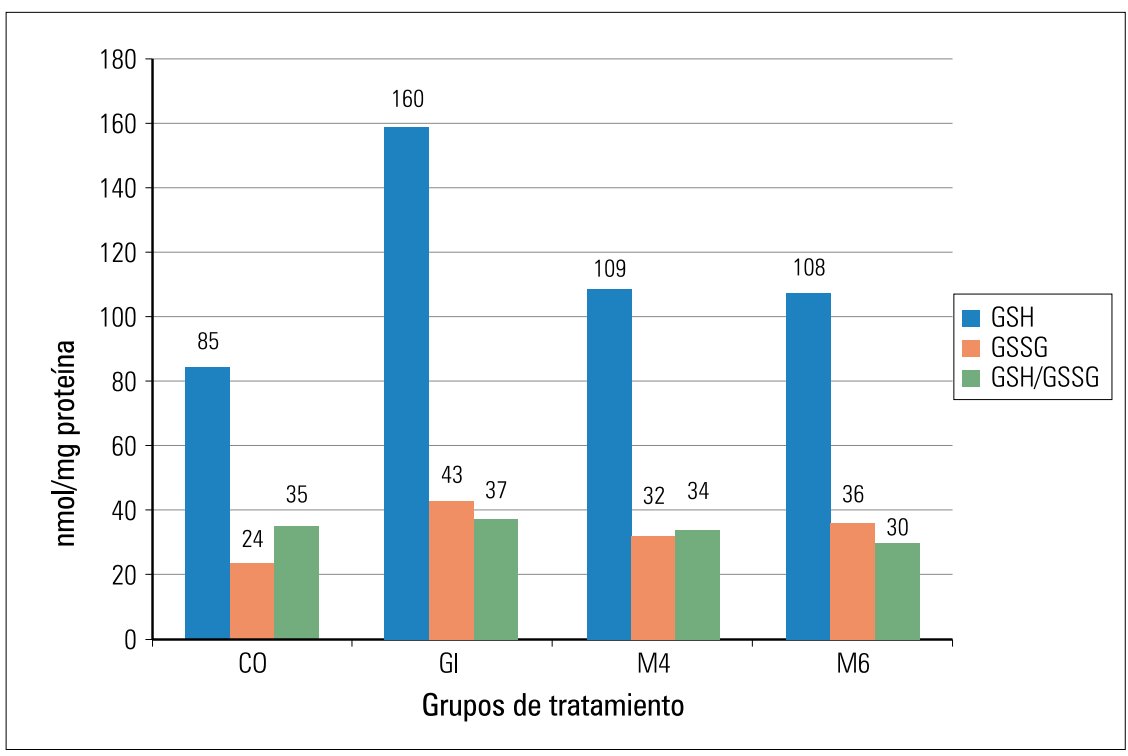

Figura 2. Niveles de GSH, GSSG y relación GSH/GSSG en homogenizado de hígado de ratas diabéticas, según grupo de tratamiento.

\section{DISCUSIÓN}

La relación cualitativa entre el nivel de glicemia, la acumulación tisular de especies de glicación avanzada (AGEs) y los diferentes matices de la patología microvascular diabética han sido objeto de extensos estudios en modelos animales. Una primera constatación, en apariencia paradójica, es que la formación de AGEs aumenta en un grado mucho mayor que el aumento de la glicemia; este hecho sugiere que incluso elevaciones moderadas en la glicemia en los diabéticos resultarían en aumentos sustanciales, no lineares, en la acumulación de AGEs ${ }^{(24)}$. La glicación es un fenómeno importante en el desgaste tisular y junto con el estrés oxidativo (EOx) forman la base de las teorías estocásticas del envejecimiento. Estos procesos están incrementados en pacientes diabéticos. Se cree que los AGEs circulantes son probablemente el resultado del catabolismo incompleto de proteínas AGEs, a cargo de los macrófagos y otras células ${ }^{(25)}$. Es así como la hiperglucemia crónica ha sido vinculada con el EOx en los pacientes diabéticos, en los cuales se ha descrito niveles elevados de marcadores de oxidación, además de asociación con el control glucémico y los AGEs.
Fahey y col, en 2001, demostraron la actividad antioxidante y anticarcinogénica del hipocótilo de la maca, debido a su contenido de isotiocianato ${ }^{(26)}$. Se ha encontrado, en 2002, que la maca presenta la capacidad de eliminar radicales libres y proteger a las células contra el daño oxidativo ${ }^{(27)}$.

Es interesante observar que en los animales tratados con maca y contro- les no hubo pérdida de peso respecto al grupo tratado con glibenclamida, lo que podría ser explicado por mayores niveles de insulina, favorecidos con el tratamiento por maca ${ }^{(28)}$. Debe agregarse que, además, durante los 40 días del tratamiento se observó que los animales de los grupos tratados con maca desarrollaban un incremento de los valores de GSH, tanto en el homogenizado de hígado y en el plasma (figura 3 y 4), con respecto al grupo CO.

La relación GSH/GSSG en condiciones fisiológicas es mayor que 100 . Con el estrés oxidativo se acumula GSSG y disminuye la relación, lo que indica cambios en el estado redox que afectan el balance de la proliferación, la diferenciación y la muerte celular.

En el presente estudio, esta relación a nivel hepático mostró valores menores de 100, lo que significa que existió una mayor proporción de la forma oxidada, probablemente producto de la actividad de GSH peroxidasa o por un deficiente sistema de recuperación de la forma reducida; esto ocurrió aún cuando los niveles de GSH reducido en el grupo M4 fue significativamente mayor al grupo CO. El mismo comportamiento fue observado en el grupo M6, pero sin significancia estadística.

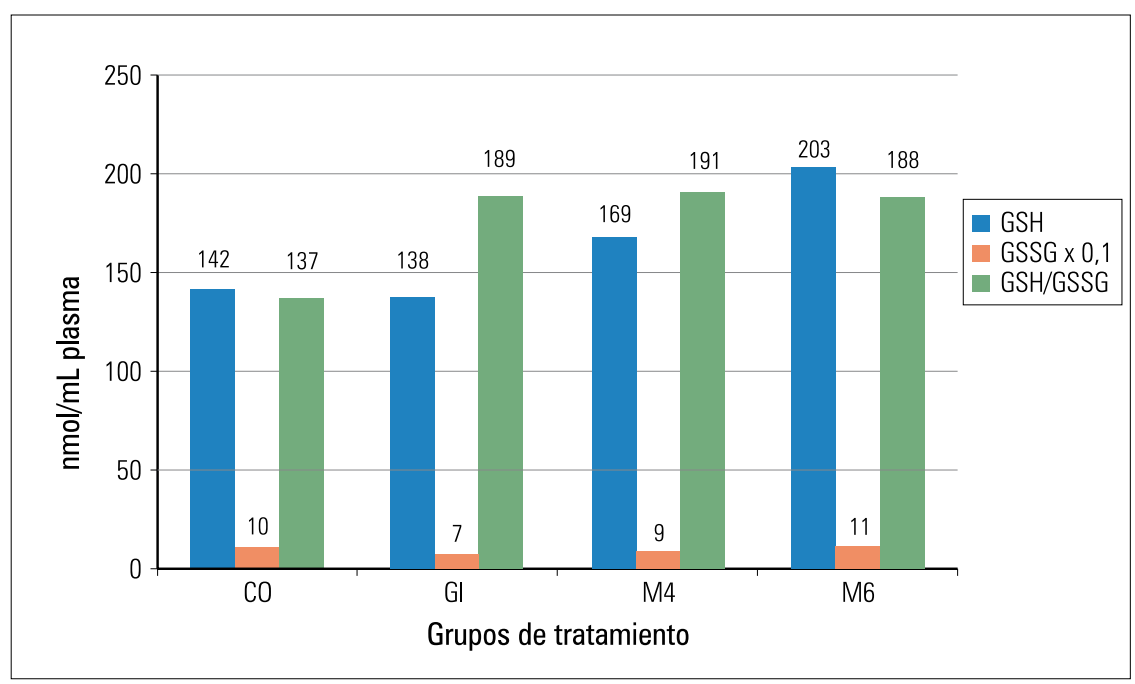

Figura 3. Niveles de GSH, GSSG y relación GSH/GSSG en plasma de ratas diabéticas, según grupo de tratamiento. 
Analizada esta relación a nivel plasmático, los resultados muestran que el glutatión circulante se encontró favorecido para la forma reducida, pues los valores de la relación GSH/GSSG estuvieron alrededor de 190 , significativamente mayor al grupo $\mathrm{CO}$.

La determinación de la relación en plasma de GSH/GSSG reflejaría los cambios que al parecer se producen en tejidos extrahepáticos. La presencia de insuficientes cantidades de GSH pone en marcha la síntesis de novo de GSH. Este tipo de síntesis se lleva a cabo a partir de los precursores del GSH y no por transformación a partir del GSSG. La finalidad de este mecanismo es restablecer los valores de GSH ${ }^{(29)}$. Otra posible explicación sería la exportación de GSH reducido de los hematíes, conociendo que tiene un poderoso sistema de recuperación de GSH, debido a la permanente agresión oxidativa de la molécula de oxígeno que debe transportar mediante la hemoglobina.

En situaciones patológicas, las células preapoptóticas y apoptóticas tienen una relación GSH/GSSG baja, incluyendo a las proteínas con grupos $-\mathrm{SH}$, con relación a las células normales, lo que evidencia el papel señalizador de los niveles disminuidos de GSH reducido o los niveles incrementados de GSSG, como parte de la regulación redox en la homeostasis celular ${ }^{(30)}$.

Se puede concluir que la administración de la harina de maca amarilla en el modelo experimental del presente estudio produjo un aumento de la relación glutatión reducido/ glutatión oxidado (GSH/GSSG) a nivel plasmático, contribuyendo a mejorar su estado redox. Estos resultados constituyen los primeros que se reportan para la administración de harina de maca amarilla en un modelo de diabetes experimental y servirán de referencia para posteriores estudios que se requiere para conocer los mecanismos de acción de dicho tubérculo en este problema de salud pública.

\section{AGRADECIMIENTOS}

A Juan Trabucco Ricaldi - Maestría en Bioquímica, Universidad Nacional Mayor de San Marcos.

A María Elena Rodrigo Rojas Maestría en Bioquímica, Universidad Nacional Mayor de San Marcos.

\section{REFERENCIAS BIBLIOGRÁFICAS}

1. Diaz D. Hiperglicemia y estrés oxidativo en el paciente diabético. Rev Cubana Invest Bioméd. 2006;25(3).

2. Moreno AL. Epidemiologia y diabetes. Rev Fac Med UNAM. 2001;44:35-7.

3. Seclén S, Leey J, Villena A, Herrera B, Menacho J, Carrasco A y col. Prevalencia de diabetes mellitus, hipertensión arterial, hipercolesterolemia y obesidad como factores de riesgo coronario y cerebrovascular en población adulta de la costa sierra y selva de Perú. Acta Med Peru. 1999;17(1):8-12.

4. Untiveros F, Nuñez O, Tapia L, Tapia G. Diabetes mellitus tipo 2 en el Hospital II Essalud - Cañete: Aspectos demográficos y clínicos. Rev Med Hered. 2004;15(1):19-23.

5. Rosado J, Mendoza V. Mini-revisión: Inflamación crónica y estrés oxidativo en la diabetes mellitus. Bioquimia. 2007;32(2):58-69.

6. Martínez M, Andrés D, Zubillaga M. Conceptos actuales del metabolismo del glutatión. Utilización de los isótopos estables para la evaluación de su homeostasis. Acta Bioquim Clin Latinoam. 2006;40(1):45-51.

7. Toescu V, Nuttall S, Martin U, Kendall M, Dunne F. Oxidative stress and normal pregnancy. Clin Endocrinol. 2002;57:609-13.

8. Viña J, Pérez C, Furukawa T, Palacín M, Viña JR Effect of oral glutathione on hepatic glutathione levels in rats and mice. Br J Nutr. 1989;62:683-91.

9. Meister A. Glutathione metabolism and its selective modification. J Biol Chem. 1988;263(33):17205-8.

10. Belda JI, Puertas J, Morell F, Romero M, Marín N, Díaz-Llopis M, Romero FJ. Determinación de los niveles de glutatión, enzimas del sistema glutatión y productos de la peroxidación lipídica en el cristalino cataratoso y en el cristalino sano. Arch Soc Esp Oftalmol. 1999;74(11).

11. Jones D, Carlson JL, Mody VC, Cai J, Lynn M, Sternberg $P$. Redox state of glutathione in human plasma. Free Rad Biol Med. 2000;28(4):625-35.

12. Cisneros $E$. La glutatión reductasa y su importancia biomédica. Rev Cubana Invest Bioméd. 1995;14(1):0-0.

13. Cisneros E, Pupo J, Céspedes E. Enzimas que participan como barreras fisiológicas para eliminar los radicales libres: III. Glutatión peroxidasa. Rev Cubana Invest Bioméd. 1997;16(1):10-5.

14. Deneke S, Fanburg B. Regulation of cellular glutathione. Am J Physiol. 1989;257:163-73.

15. Cuentas R, De la Cruz L, Hernández G, Mateo I, Castañeda C, Ibáñez L, Ramos E. Evaluación del efecto antioxidante de hojas de Lepidium peruvianum Chacón, "maca”. Rev Horiz Méd. 2008;8(1):45-55.

16. Bianchi A. Maca Lepidium meyenii. Boletín Latinoamericano y del Caribe de plantas medicinales y aromáticas. 2003;2(3):26-44.
17. Castaño M. Maca (Lepidium peruvianum Chacon): composición química y propiedades farmacológicas. Clin Cancer Res. 2006;12(5):1447-53.

18. Suárez S, Oré R, Arnao I, Rojas L, Trabucco J. Extracto acuoso de Lepidium meyenii Walp (maca) y su papel como adaptógeno, en un modelo animal de resistencia física. An Fac med. 2009;70(3):181-5.

19. Cicero AFG, Bandieri E, Arletti R. Lepidium meyenii Walp improves sexual behaviour in male rats independently from its action on spontaneous locomotor activity. J. Ethnopharmacol. 2001;75:225-9.

20. Muhamma I, Zhao J, Dunbar C, Khan IA. Constituents of Lepidium meyenii "maca". Phytochemistry. 2002;59:105-10.

21. Vit $P$, de Jesús R, Gudiño M, Jacob T. Inducción de cataratas experimentales en ratas diabetizadas con estreptozotocina. Rev Fac de Farma Univ de los Andes Venezuela. 2002;43(1):15-8.

22. National Research Council. Guide for the care and use of laboratory animals. Washington, DC: National Academy Press; 1996.

23. Boyne A, Ellman G. Assay for glutathione. Anal Biochem. 1972;46:639-53.

24. Gugliucci A. Glicación de proteinas: rol protagónico de la hiperglicemia en las complicaciones crónicas de la diabetes mellitus. Rev Med Uruguay. 2000;16:58-75.

25. Bucala R, Vlassara H. Advanced glycosylation end products in diabetic renal and vascular disease. A J Kidney Dis. 1995;26:875-88.

26. Fahey JW, Zalcmann AT, Talalay P. The chemical diversity and distribution of glucosinolates and isothiocyanates among plants. Phytochemistry. 2001;56:5-51.

27. Oré R, Huerta D, Sandoval M, Valdivieso R, Rodrigo M, Durand J. Actividad antioxidante in vitro de dos extractos de raices de ecotipo amarillo de Lepidium meyenii Walp (maca). An Fac med. 2009;70 Supl 1.

28. Rodrigo M, Valdiviezo R, Suárez S, Oriondo R, Oré R. Disminucion del daño oxidativo y efecto hipoglicemiante de la maca (Lepidium meyenii Walp) en ratas con diabetes inducida por estreptozotocina. An Fac med. 2011;72(1):7-11.

29. Di Toro, C. Glutatión como agente protector endogéno en las enfermedades degenerativas y psiquiátricas. Psicofarmacología. 2003;22.

30. Aviram M. Interaction of oxidized low density lipoprotein with macrophages in atherosclerosis and antiatherogenicity of antioxidants. Eur J Clin Chem Clin Biochem. 1996;34:599-608.

Trabajo ganador del premio al Mejor trabajo del Docente menor de 40 años, de la Asociación de Exalumnos Sanfernandinos de la Peruvian American Medical Society, de las IX Jornadas Cientificas Sanfernandinas, XII Jornadas de Investigación en Salud, XIX Jornadas Sanfernandinas Estudiantiles, Facultad de Medicina, UNMSM, setiembre 2010.

Correspondencia:

Ruth Cisneros Ch.

ruthicisneros@yahoo.com.mx 